\title{
Chemistry of Unsaturated Group 6 Metal Complexes with Bridging Hydroxy and Methoxycarbyne Ligands. Part 1. Synthesis, Structure and Bonding of 30-Electron Complexes.
}

\author{
M. Esther García, Daniel García-Vivó, Miguel A. Ruiz,* Santiago Alvarez, ${ }^{\dagger}$ and \\ Gabriel Aullón ${ }^{\dagger}$
}

Departamento de Química Orgánica e Inorgánica/IUQOEM, Universidad de Oviedo, E-33071 Oviedo, Spain. ${ }^{\dagger}$ Departamento de Química Inorgánica, Universidad de Barcelona, E-08028 Barcelona, Spain.

\section{Supporting Information}


Table S1. Selected atomic charges in the complexes $\mathbf{2}$ to $\mathbf{7}$

\begin{tabular}{|c|c|c|c|c|c|c|c|c|c|c|}
\hline \multirow{3}{*}{ Atom } & \multicolumn{10}{|c|}{ Compound } \\
\hline & \multicolumn{2}{|c|}{2} & \multicolumn{2}{|c|}{6} & \multicolumn{2}{|c|}{$\mathbf{A}$} & \multicolumn{2}{|c|}{3} & \multicolumn{2}{|c|}{7} \\
\hline & Mulliken & NPA & Mulliken & NPA & Mulliken & NPA & Mulliken & NPA & Mulliken & NPA \\
\hline $\operatorname{Mo}(1)$ & -0.089 & -0.222 & -0.012 & -0.060 & -0.072 & -0.131 & 0.050 & 0.024 & 0.156 & 0.118 \\
\hline $\operatorname{Mo}(2)$ & -0.091 & -0.225 & -0.020 & -0.124 & -0.072 & -0.131 & 0.063 & -0.030 & 0.156 & 0.119 \\
\hline $\mathrm{P}$ & $\begin{array}{l}0.282 \\
0.286\end{array}$ & $\begin{array}{l}0.960 \\
0.960\end{array}$ & $\begin{array}{l}0.279 \\
0.281\end{array}$ & $\begin{array}{l}0.996 \\
0.997\end{array}$ & 0.247 & 0.851 & 0.267 & 0.919 & 0.289 & 0.971 \\
\hline $\mathrm{C}_{\text {carbyne }}$ & & & -0.020 & 0.266 & & & -0.019 & 0.235 & $\begin{array}{l}-0.028 \\
-0.028\end{array}$ & $\begin{array}{l}0.264 \\
0.264\end{array}$ \\
\hline $\mathrm{O}_{\text {methoxycarbyne }}$ & & & -0.028 & -0.497 & & & -0.424 & -0.513 & $\begin{array}{l}-0.402 \\
-0.402\end{array}$ & $\begin{array}{l}-0.493 \\
-0.492\end{array}$ \\
\hline $\mathrm{C}_{\text {carbonyl }}$ & 0.140 & 0.421 & & & $\begin{array}{l}0.162 \\
0.161\end{array}$ & $\begin{array}{l}0.409 \\
0.409\end{array}$ & 0.135 & 0.416 & & \\
\hline $\mathrm{O}_{\text {carbonyl }}$ & -0.382 & -0.531 & & & $\begin{array}{l}-0.448 \\
-0.448\end{array}$ & $\begin{array}{l}-0.592 \\
-0.593\end{array}$ & -0.385 & -0.532 & & \\
\hline $\mathrm{C}_{\text {methoxy }}$ & & & -0.225 & -0.304 & & & -0.207 & -0.301 & $\begin{array}{l}-0.227 \\
-0.227\end{array}$ & $\begin{array}{l}-0.306 \\
-0.306\end{array}$ \\
\hline
\end{tabular}


Table S2. Selected KS molecular orbitals for compound 2

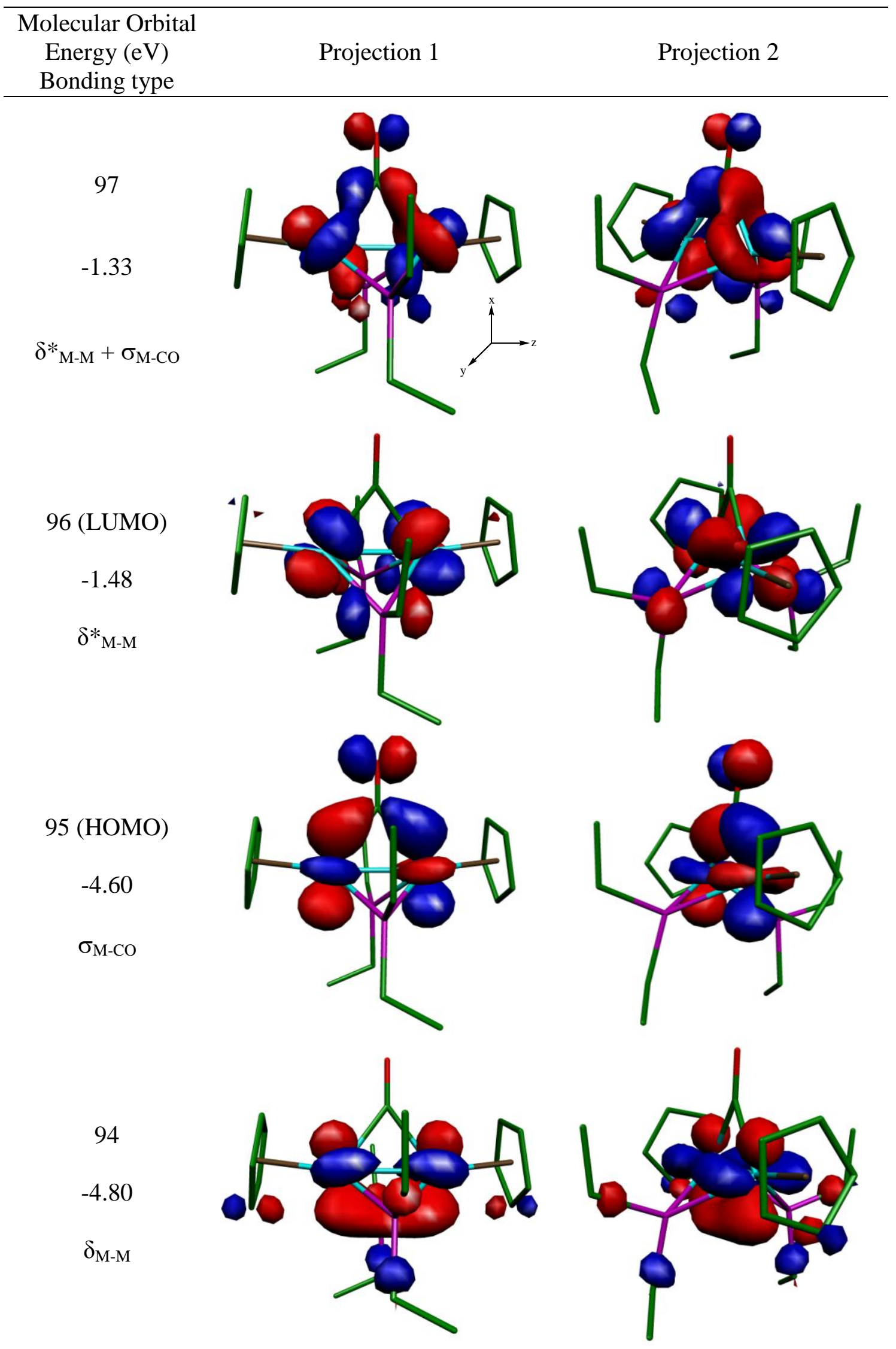




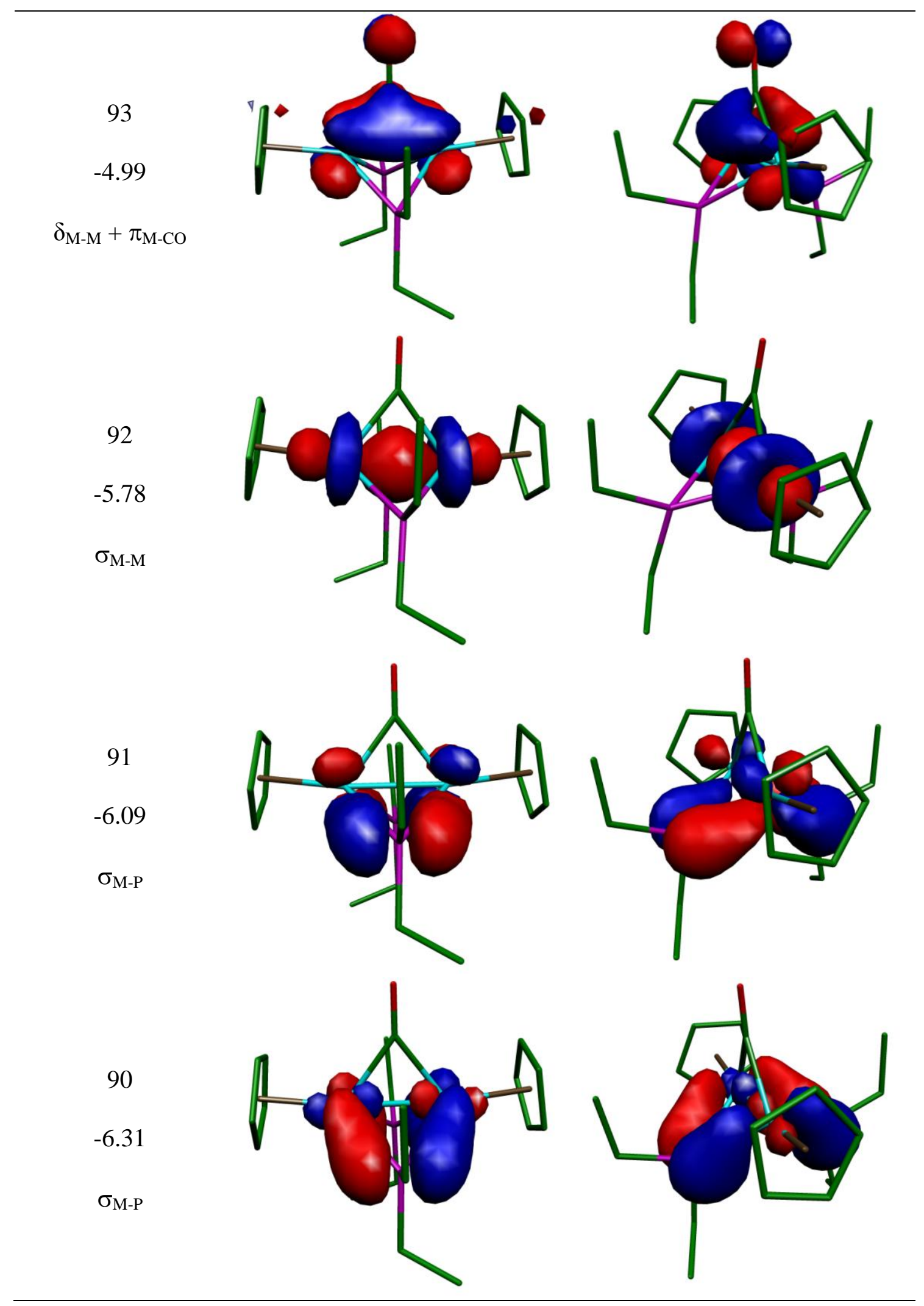


Table S3. Selected KS molecular orbitals for the cation in compound 6

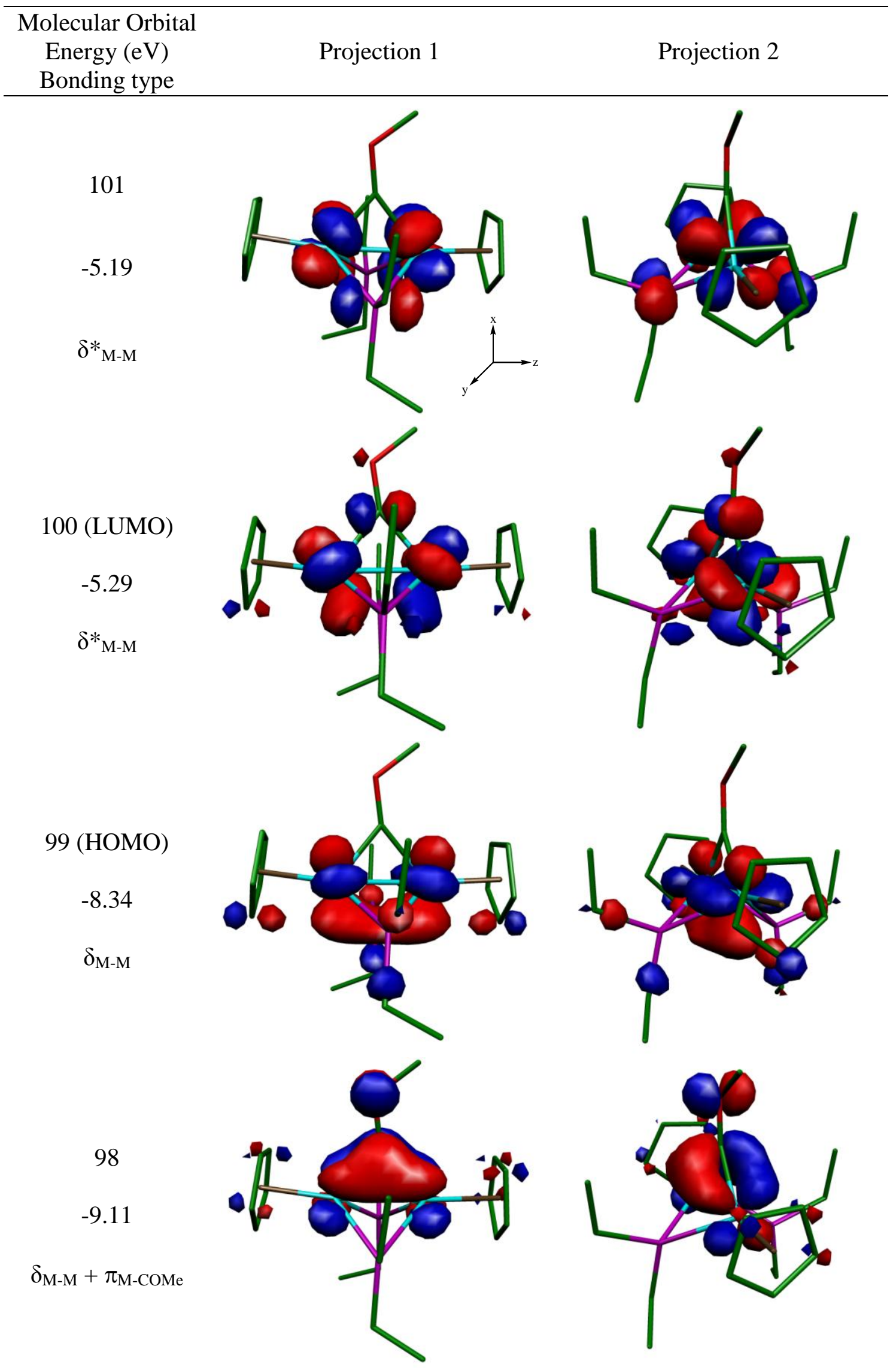




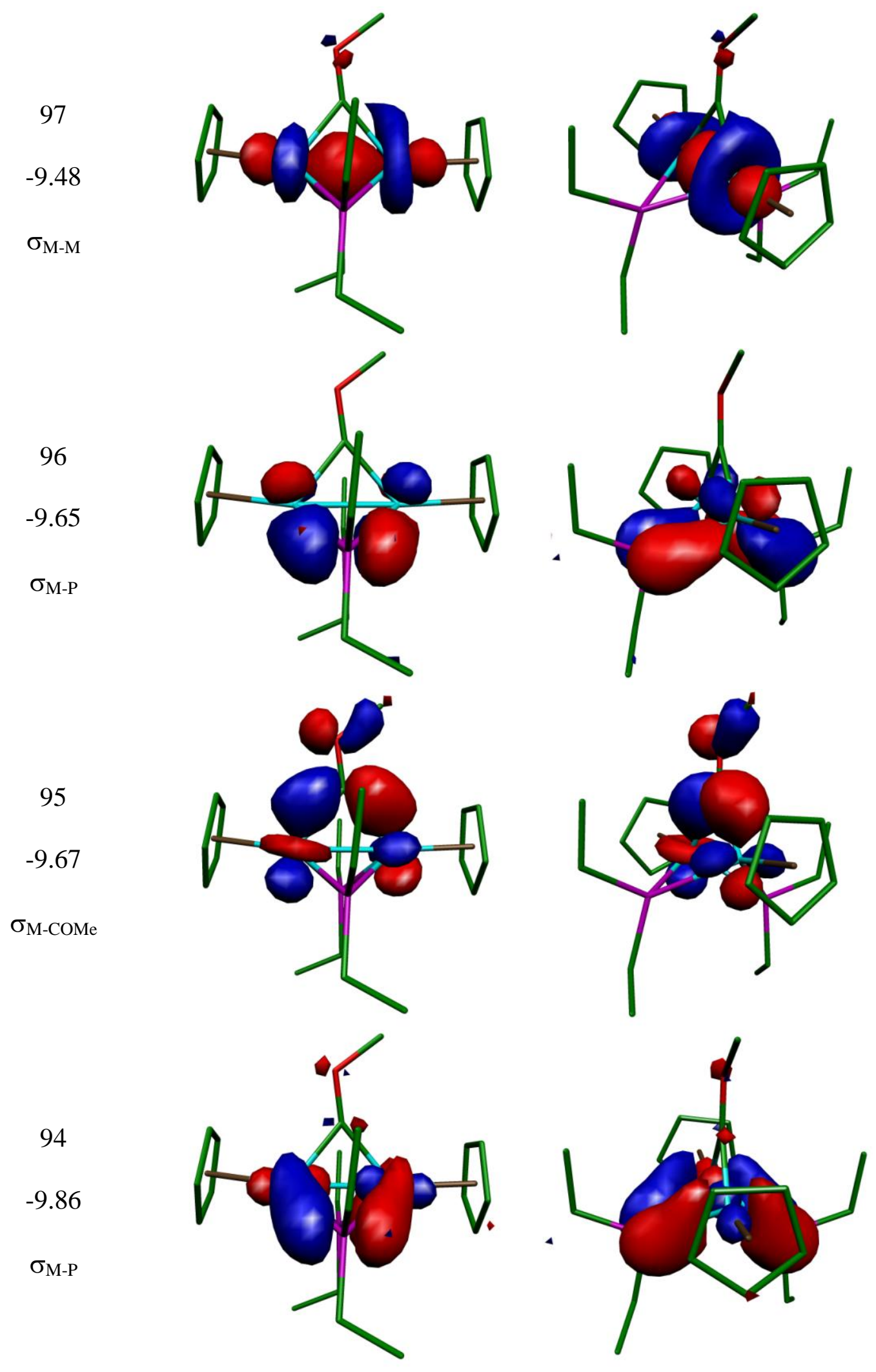




$$
\begin{aligned}
& =100 \\
& \therefore 1000
\end{aligned}
$$


Table S4. Selected KS molecular orbitals for the anionic complex A.

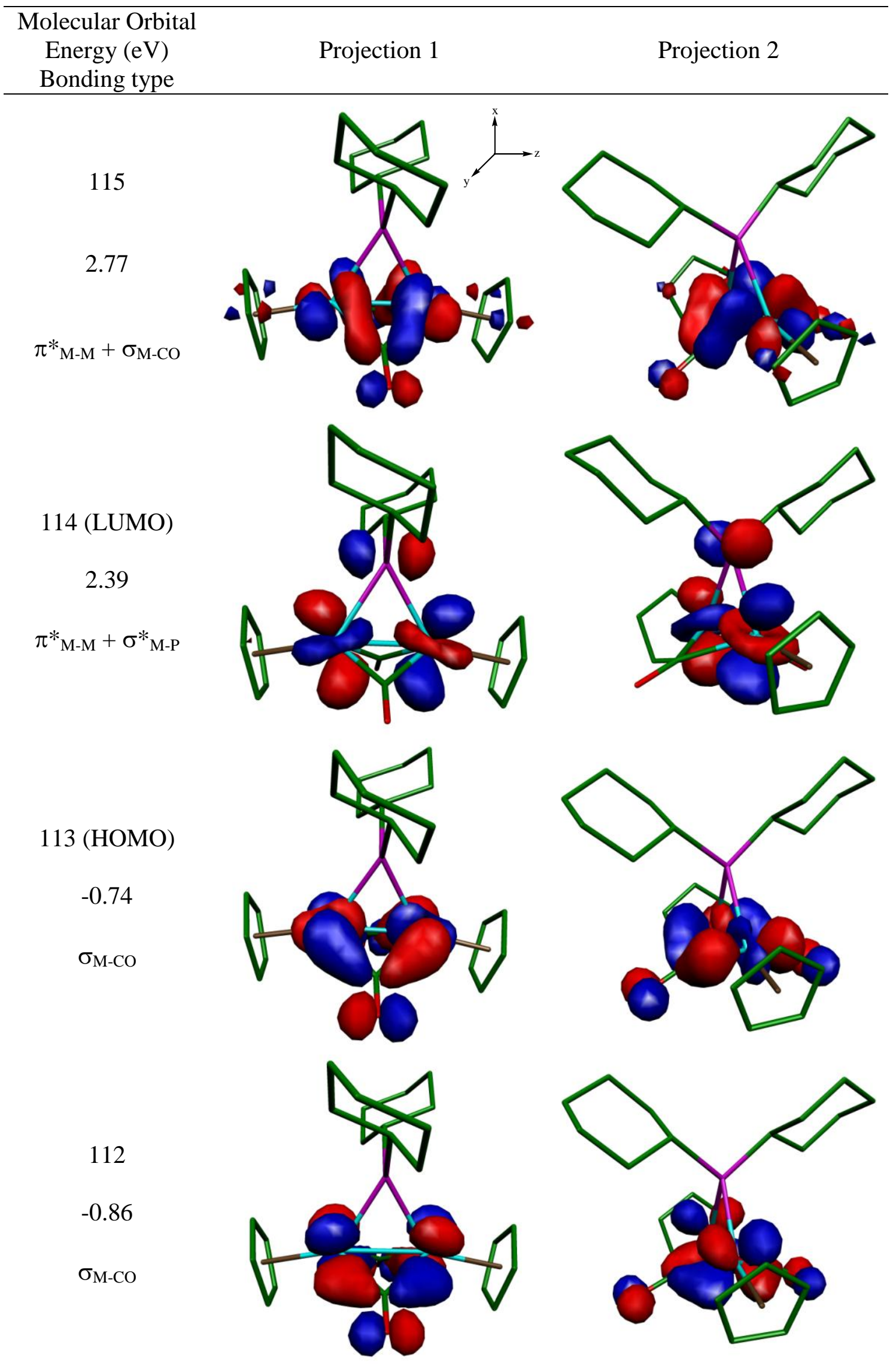




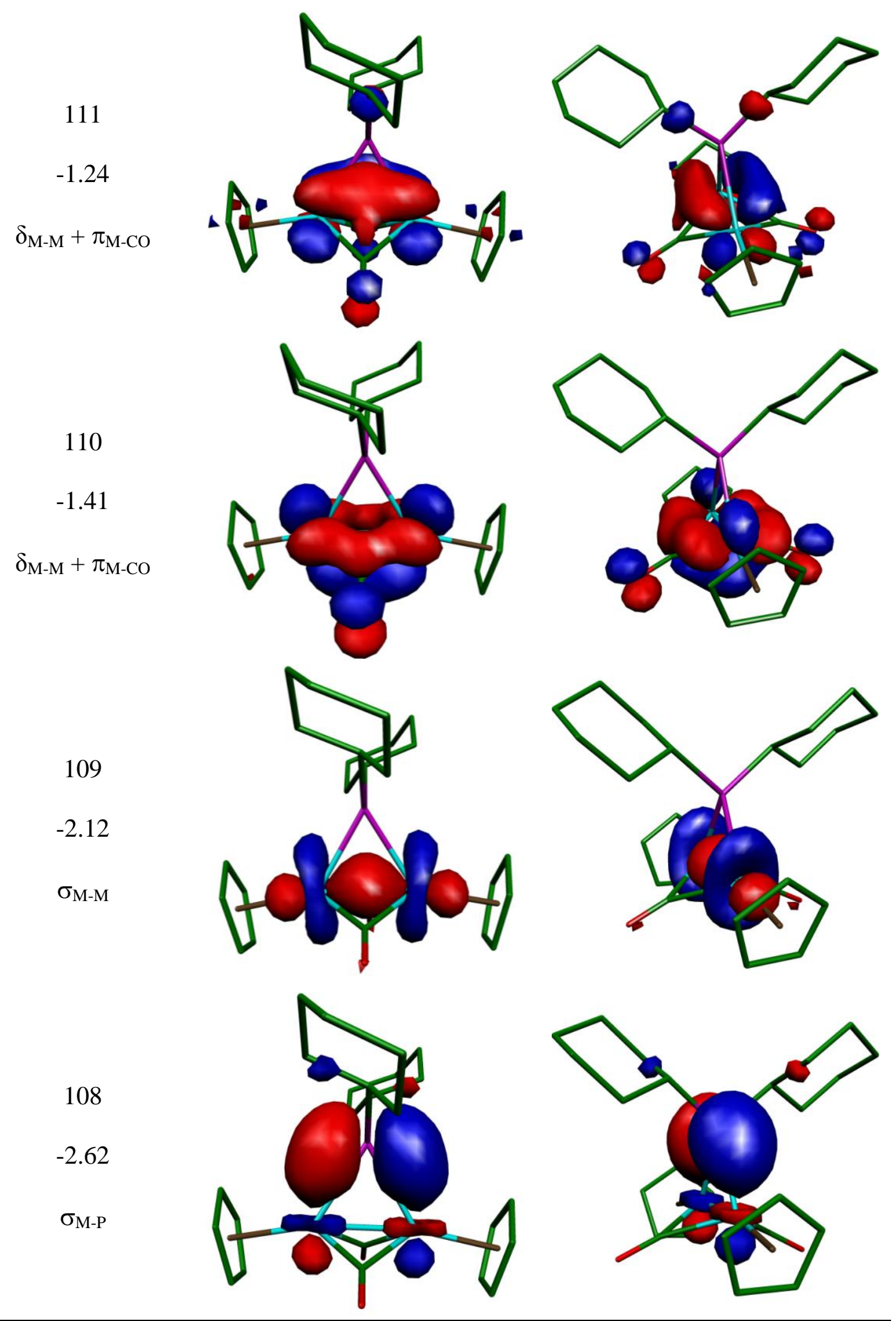


Table S5. Selected KS molecular orbitals for compound 3.

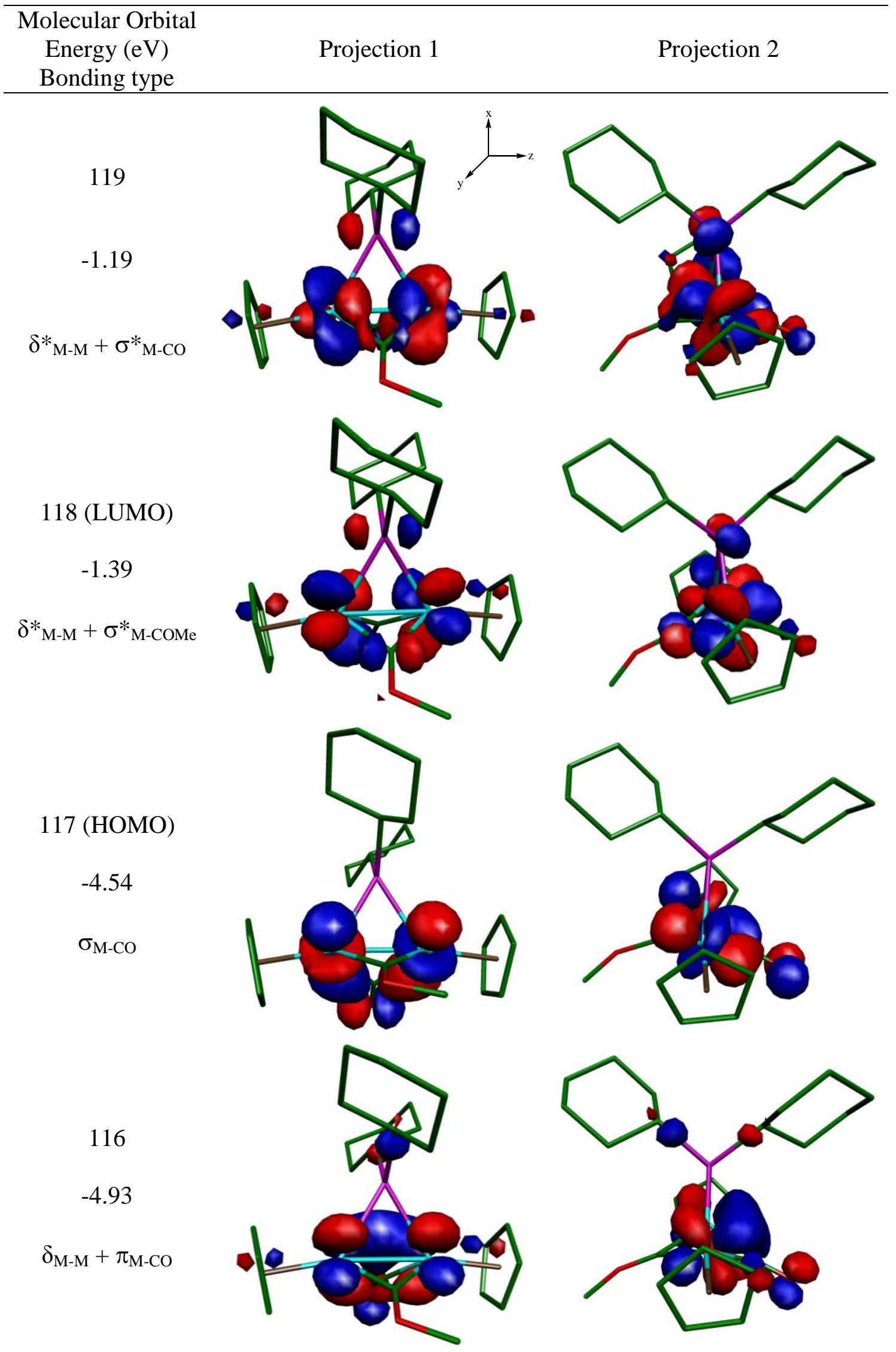




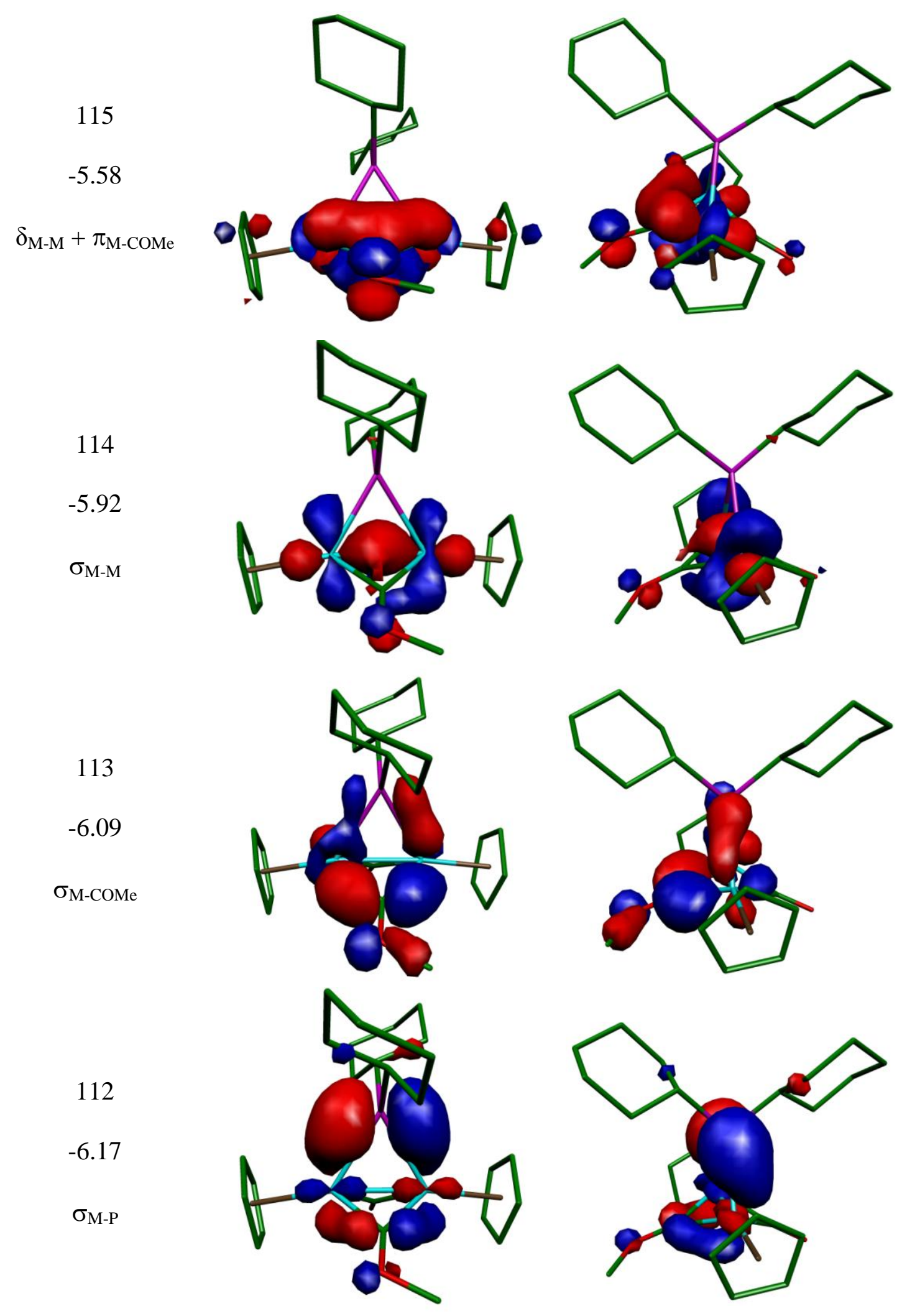




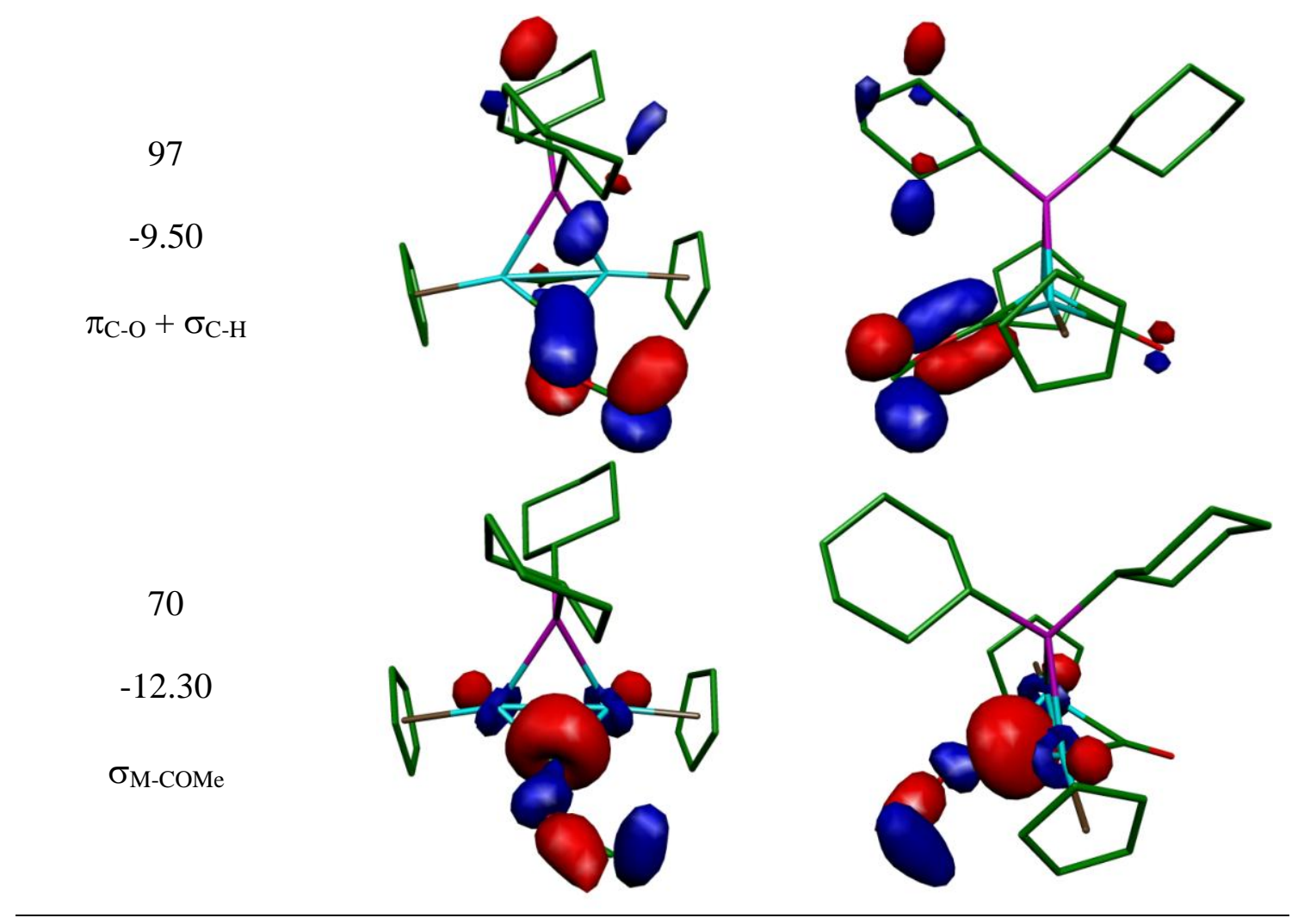


Table S6. Selected KSmolecular orbitals for the cation in compound 7.

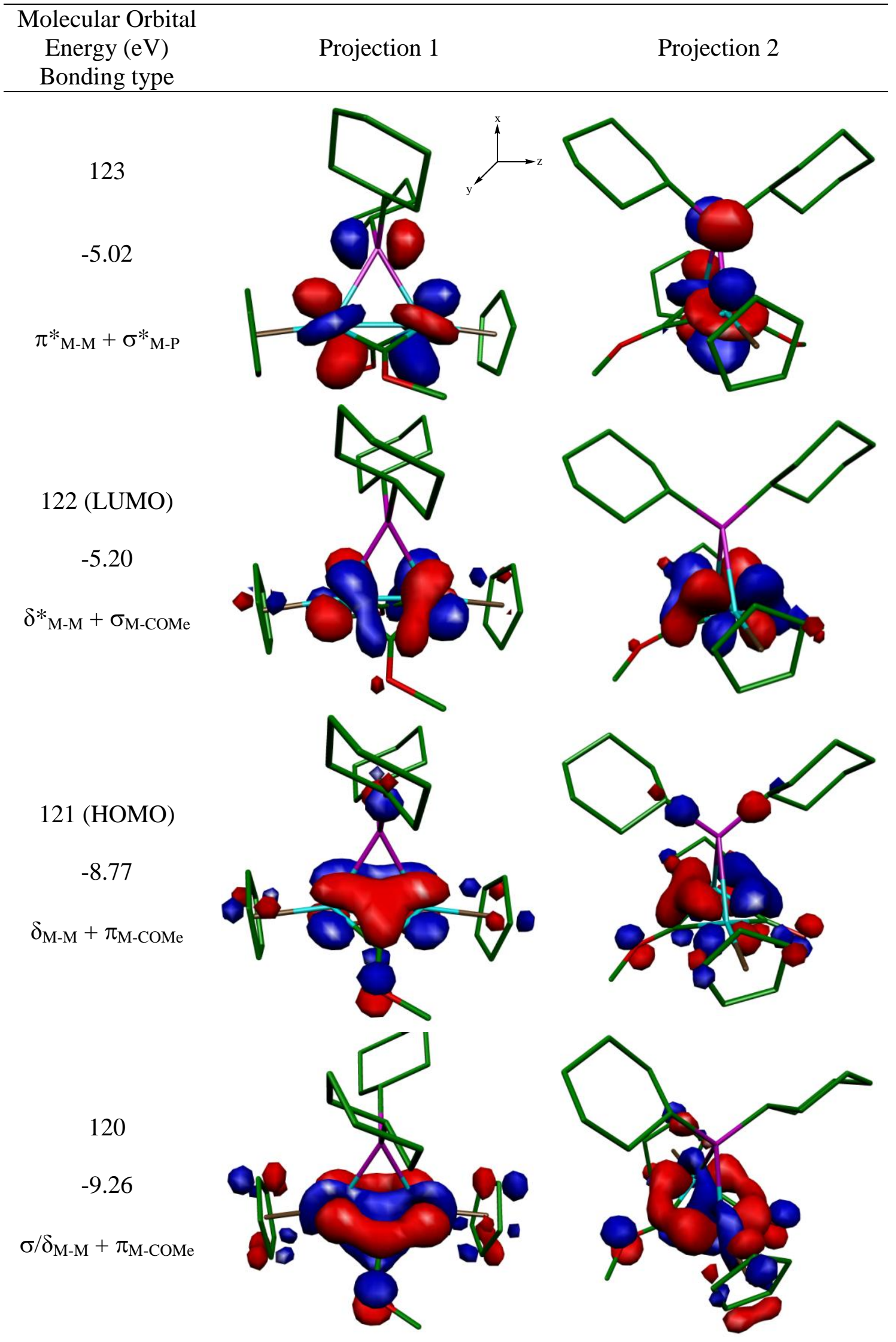




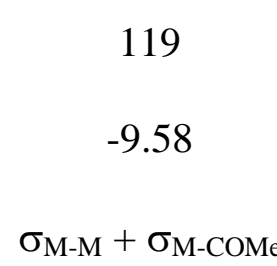

118

$-9.60$

$\sigma_{M-P}$

117

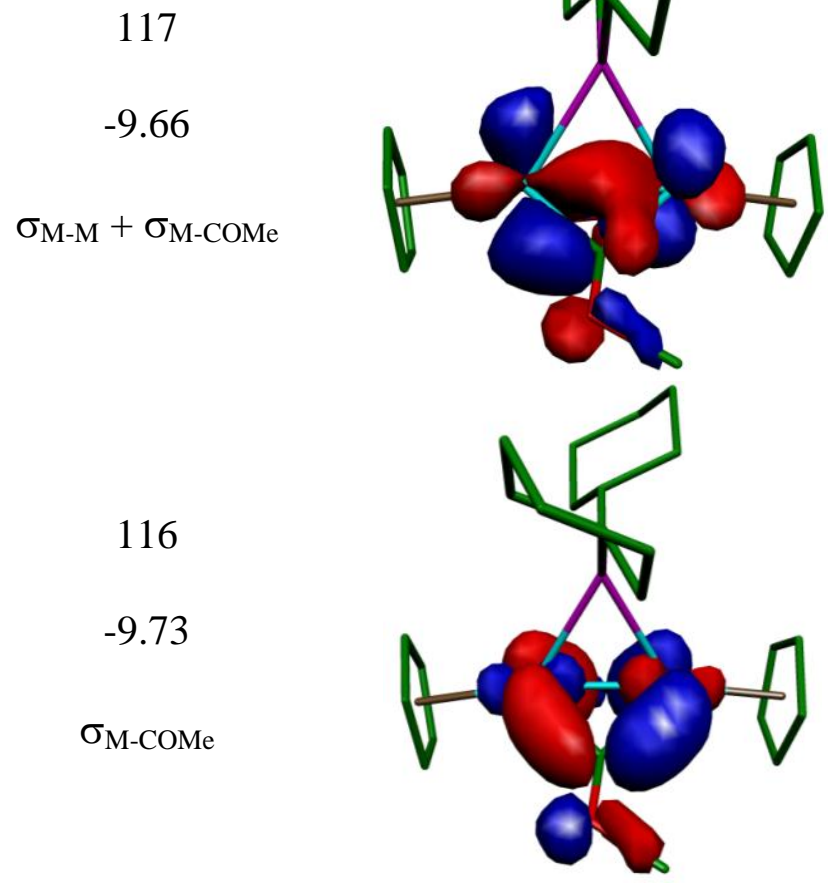

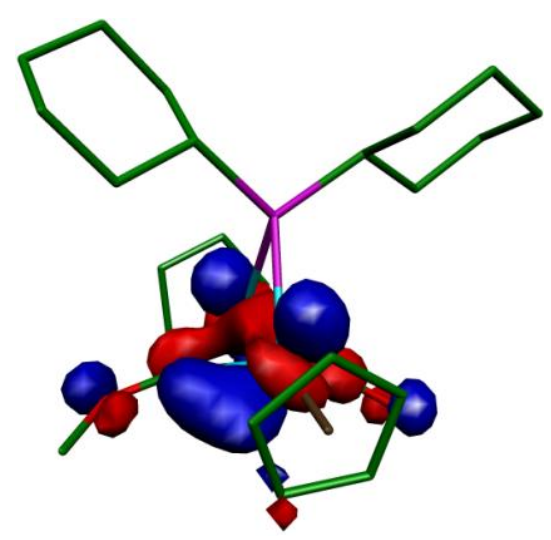
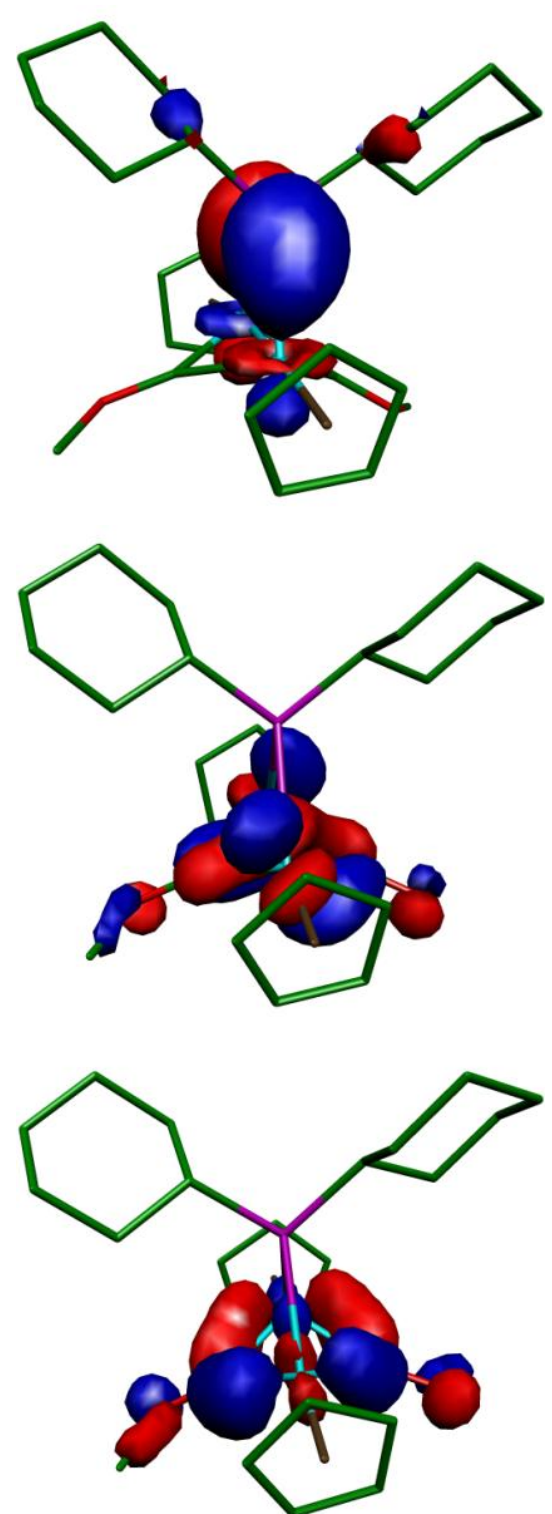


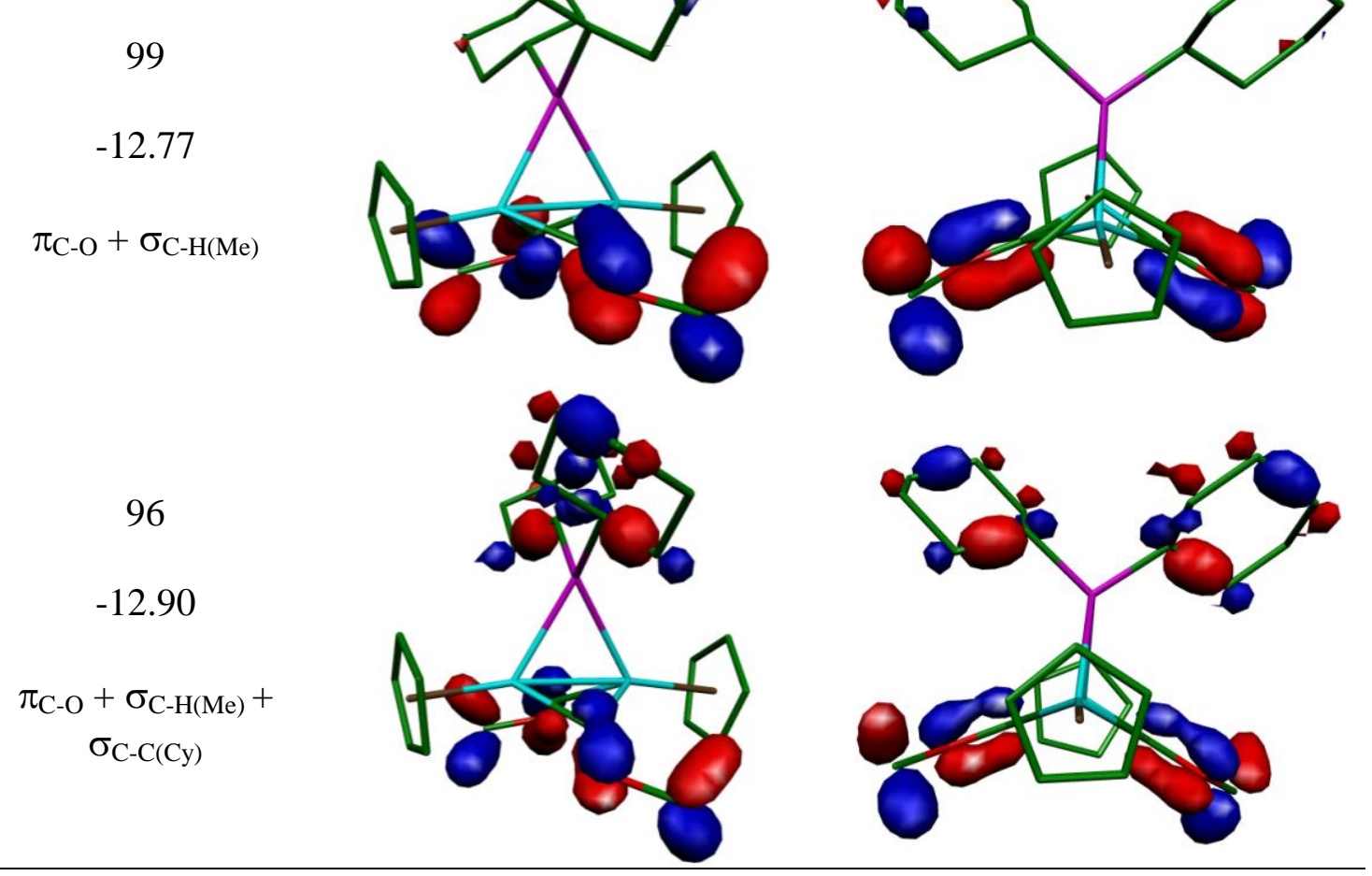




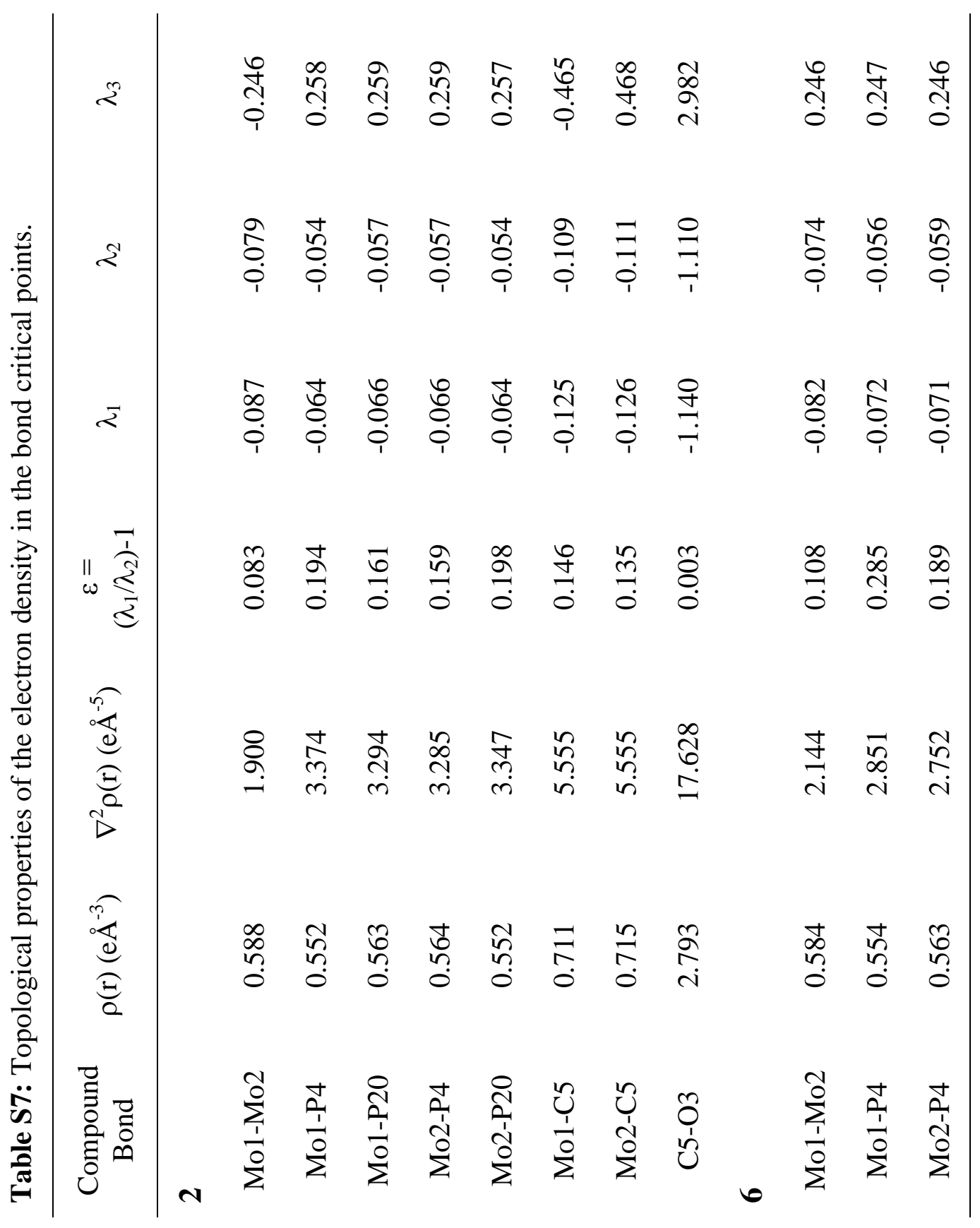




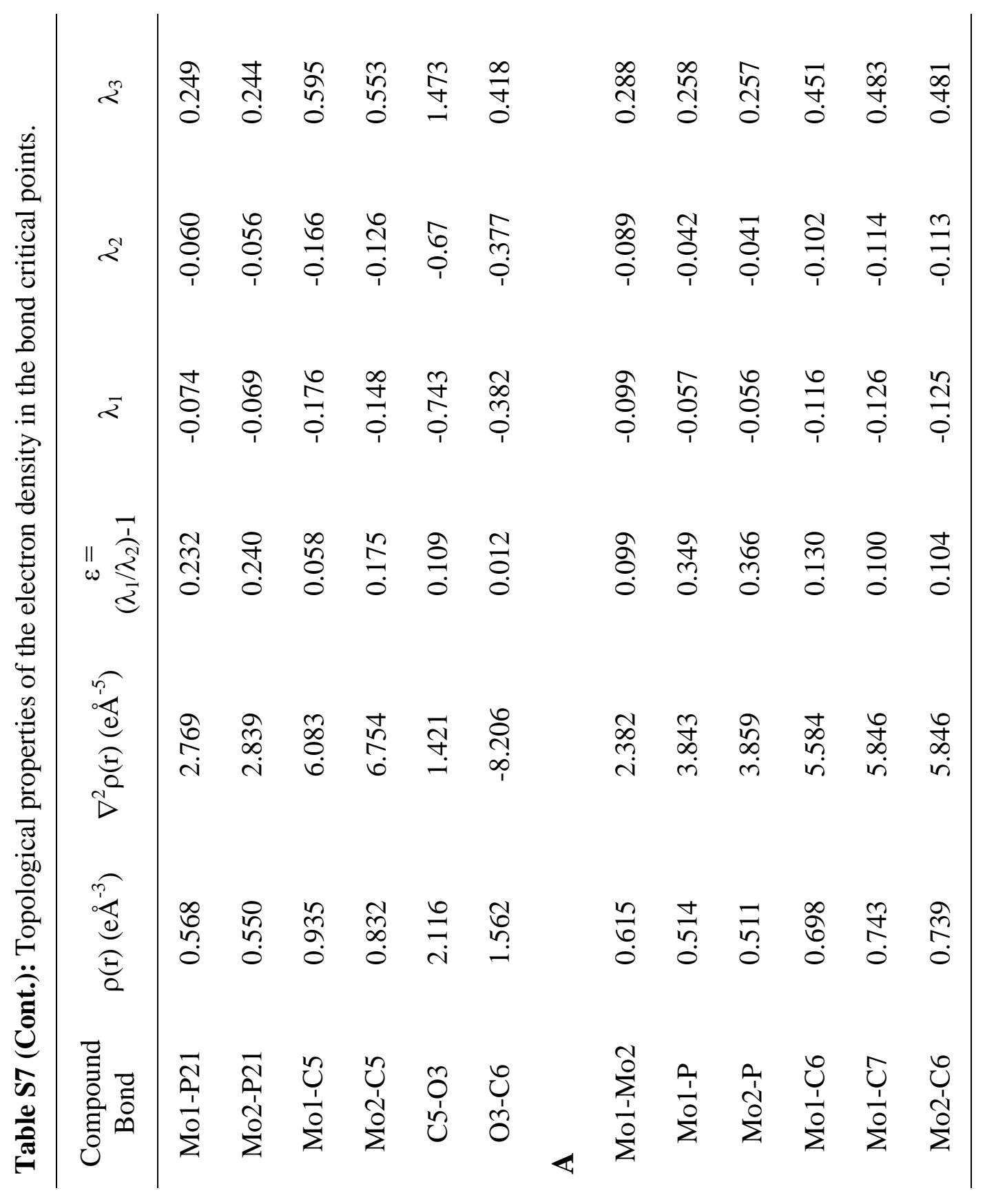




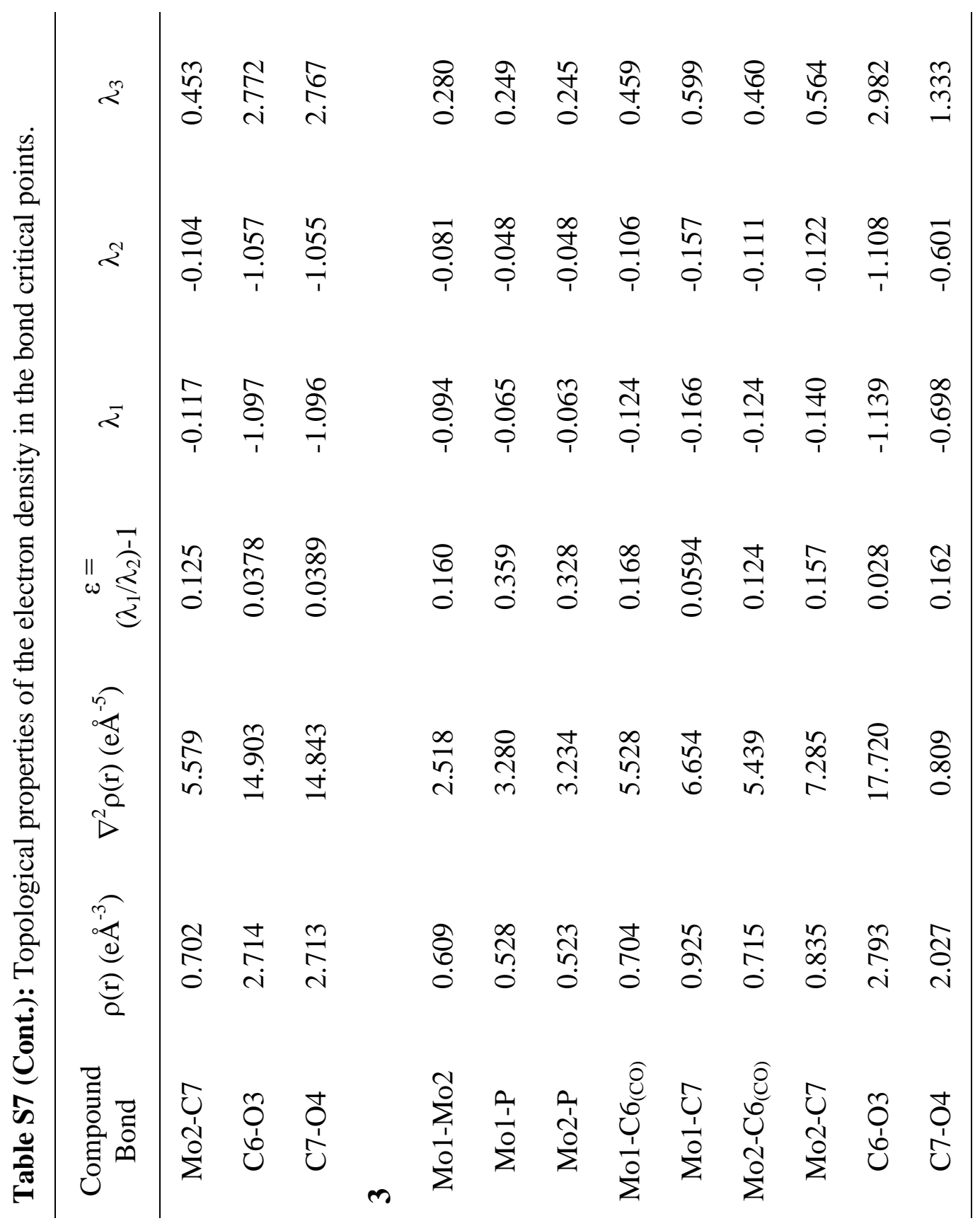




\begin{tabular}{|c|c|}
\hline & 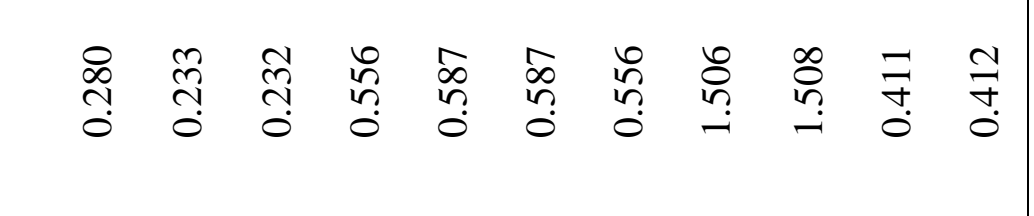 \\
\hline & 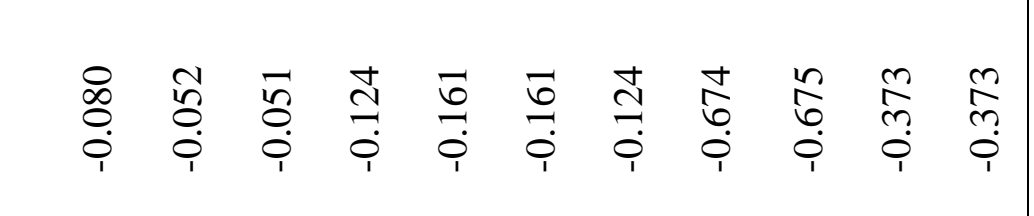 \\
\hline & 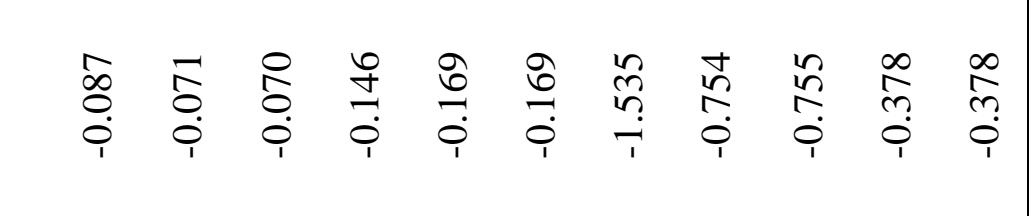 \\
\hline & 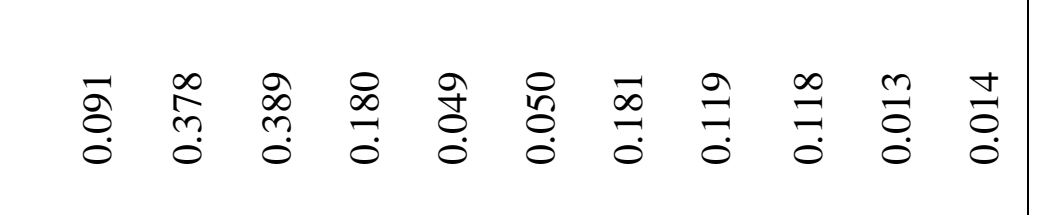 \\
\hline & 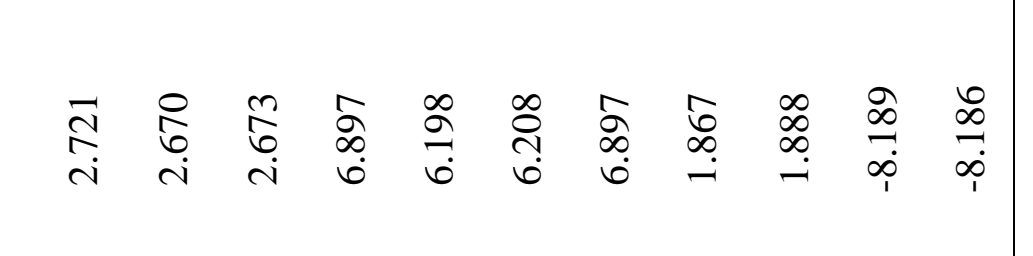 \\
\hline & 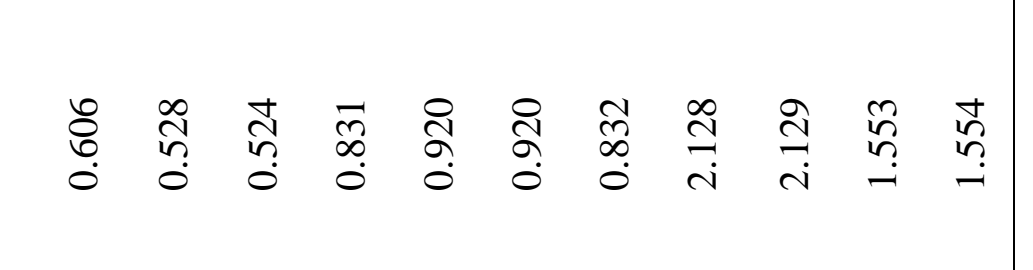 \\
\hline & 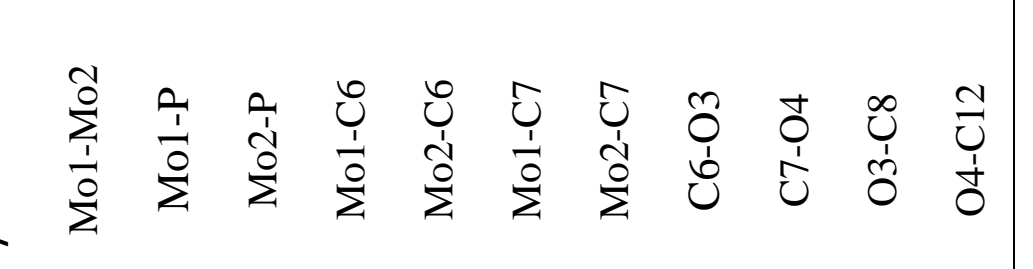 \\
\hline
\end{tabular}

